\title{
Ultrawide-Band Synthetic Aperture Radar for Detection of Unexploded Ordnance: Modeling and Measurements
}

\author{
Anders Sullivan, Member, IEEE, Raju Damarla, Member, IEEE, Norbert Geng, Member, IEEE, \\ Yanting Dong, Student Member, IEEE, and Lawrence Carin, Senior Member, IEEE
}

\begin{abstract}
Electromagnetic (EM) scattering from subsurface unexploded ordnance (UXO) is investigated both theoretically and experimentally. Three EM models are considered: the multilevel fast multipole algorithm (MLFMA), the method of moments (MoM), and physical optics (PO). The relative accuracy of these models is compared for several scattering scenarios. Moreover, the model results are compared to data measured by an experimental synthetic-aperture radar (SAR) system. SAR images have been generated for subsurface UXO targets, in particular $155-\mathrm{mm}$ shells. We compare SAR images from the measured data with theoretical images produced by the MoM and PO simulations, using a standard back-projection imaging technique. In addition to such comparisons with measurement, we consider additional buried-UXO scattering scenarios to better understand the underlying wave phenomenology.
\end{abstract}

Index Terms-Buried object detection, ground-penetrating radar, synthetic aperature radar, ultrawide-band (UWB) radar.

\section{INTRODUCTION}

$\mathbf{T}$ HE electromagnetic (EM) characterization of surface and subsurface targets has been of interest to researchers for many years [1]-[11]. Recent development of wide-band high-resolution synthetic aperture radar (SAR) technology has shown that it may be possible to detect targets buried close to the ground surface over very large open areas [12]-[15], in a high standoff mode. In general, there are two limiting factors influencing the practicality of using wide-band SAR for wide-area target detection. First, the presence of strong ground clutter due to roughness, soil inhomogeneities, and foliage may limit the radar's ability to resolve the target from clutter. Likewise, target depth can also play a major role. For deeply buried targets, the incident wave may experience significant attenuation as it penetrates the lossy soil. Consequently, while wide-band SAR detection of targets close to the ground surface is generally considered to be feasible, detection of targets buried more than $1 \mathrm{~m}$ may be possible only in low-loss soils and/or for large targets [16]. In this paper, our model assumes a

\footnotetext{
Manuscript received September 2, 1999; revised March 14, 2000. This work was supported by the U.S. Department of Defense Strategic Environmental Research and Development Program (SERDP).

A. Sullivan and R. Damarla are with the Army Research Laboratory, Adelphi, MD 20783 USA.

N. Geng is with the Institute for Microwaves and Electronics, University of Karlsruhe, 76128 Karlsruhe, Germany.

Y. Dong and L. Carin are with the Department of Electrical and Computer Engineering, Duke University, Durham, NC 27708-0291 USA.

Publisher Item Identifier S 0018-926X(00)09375-3.
}

flat air-ground interface so we concentrate exclusively on the effects of target depth and orientation, as a function of sensor parameters. The dispersion and loss associated with the soil are modeled rigorously.

In the work presented here, we consider three models for simulating EM scattering from conducting unexploded ordnance (UXO): 1) a method-of-moments (MoM) analysis for arbitrary perfectly conducting targets in a layered medium, with the lossy dispersive layers representing the typical layered character of many soils; 2) a multilevel fast multipole algorithm (MLFMA) [17] model for electrically large conducting targets above or embedded within a lossy half-space; and 3) a physical optics (PO) model [18] for perfectly conducting UXO above or below a half space. In addition to addressing this scattering problem numerically, SAR images from UXO are presented for data collected with the U.S. Army Research Laboratory BoomSAR, Adelphi, MD [15], [19]. Comparisons are made between measured and computed SAR images, the latter simulated via the MoM and PO forward solvers. In addition to addressing the model accuracy through comparisons to measurements, we subsequently use the models to examine variation of the physical parameters to determine how such impact the SAR image.

The remainder of the text is organized as follows. In Section II, we summarize the numerical models developed for simulating wide-band scattering from surface and subsurface UXO and briefly describe the experimental SAR system employed in the measurements. A comparison between results from the three models is presented in Section III. We also perform comparisons between computed and measured SAR imagery, followed by numerical experiments to address the variation of the target signature as a function of variable physical parameters. The work is summarized and conclusions drawn in Section IV.

\section{Theoretical Models And Measurement System}

\section{A. MoM and MLFMA Models}

MoM [20], [21] and MLFMA [17], [22], [26] applied here have been discussed elsewhere. We, therefore, provide only a summary of the principal issues involved in such models. Our MoM and MLFMA models both solve a combined-field integral equation (CFIE) [27], by employing triangular-patch basis functions [28]. The MoM analysis is applicable to arbitrarily shaped perfectly conducting targets in an arbitrary layered medium, while the MLFMA is restricted to the half-space case. For both problems a principal challenge involves computation 


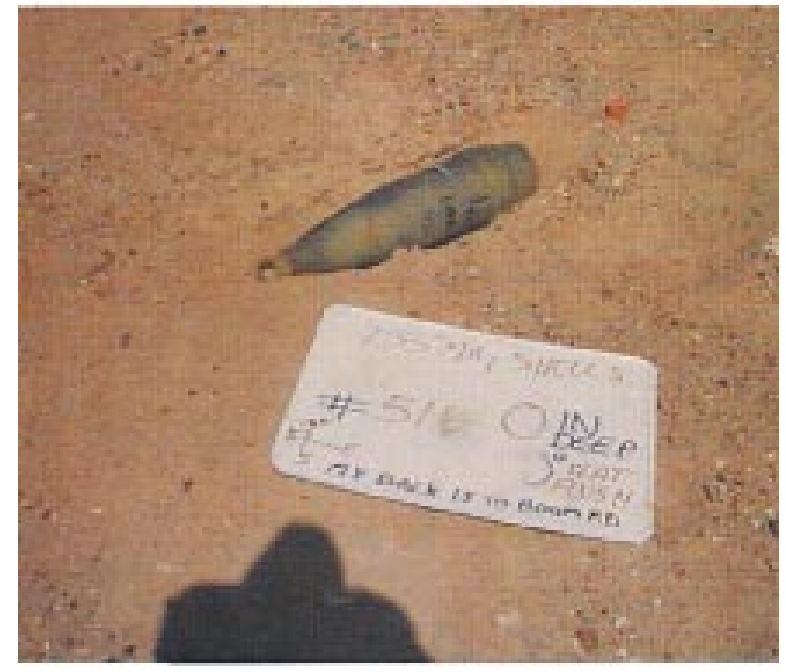

Fig. 1. Photograph of a 155-mm shell.

of the dyadic Green's function, each component of which involves Sommerfeld integrals. These integrals are solved here via the method of complex images [29]. In the MoM one computes an $N \times N$ matrix, for $N$ expansion functions, with each matrix component involving a rigorous evaluation of the dyadic Green's function. In the MLFMA analysis, expansion and testing functions are partitioned into a set of multilevel clusters, with the "far" (expansion function)-(testing function) interactions treated efficiently within the MLFMA construct [17], [26]. For this portion of the model the dyadic half-space Green's function is evaluated approximately in terms of a set of images in real space [17], [22]. However, for basis and testing functions that are sufficiently close (typically half a wavelength or less) the interactions are treated rigorously, through application of the complex-image technique.

\section{B. PO Solution}

While the MLFMA is more efficient than the MoM for large $N$, both of these models are computationally expensive for electrically large targets. We have, therefore, also developed an approximate PO solution [18] — this significantly more efficient numerically than the MoM and MLFMA models. As applied here, the UXO is partitioned into a set of triangular sections, analogous to the triangular-patch basis functions applied in the MoM and MLFMA. The surface currents on all triangles in the lit region are approximated via the $\mathrm{PO}$ approximation, with the currents on all unlit triangles set to zero. The far-zone scattered fields from these approximate currents are computed through a rigorous use of the asymptotic half-space Green's function. Concerning the incident fields used to compute the PO currents, all reflection and transmission at the half-space interface is accounted for rigorously (although multiple interactions between the target and soil interface are neglected).

\section{Experimental System}

The U.S. Army Research Laboratory has developed an experimental time-domain SAR system, with instantaneous bandwidth covering 50-1200 MHz. In this system, four horn antennas are placed atop a telescoping boom lift capable of moving

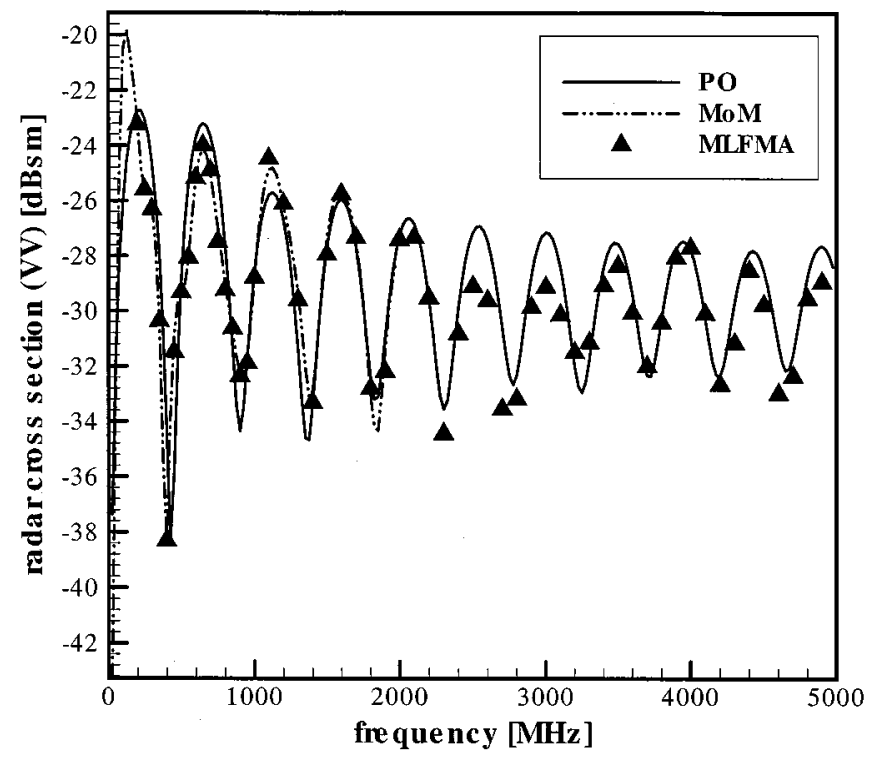

Fig. 2. Backscattered radar cross section for a $155-\mathrm{mm}$ shell buried $2.54 \mathrm{~cm}$ beneath the soil surface, with the lossy dispersive soil characterized by Yuma $5 \%$ [15]. The excitation plane wave is incident $30^{\circ}$ from grazing, propagating 45 from the target axis [see Fig. 6(b)]. Results are computed from MoM, MLFMA, and PO. The incident field is vertically polarized and we consider the VV-polarized backscattered fields.

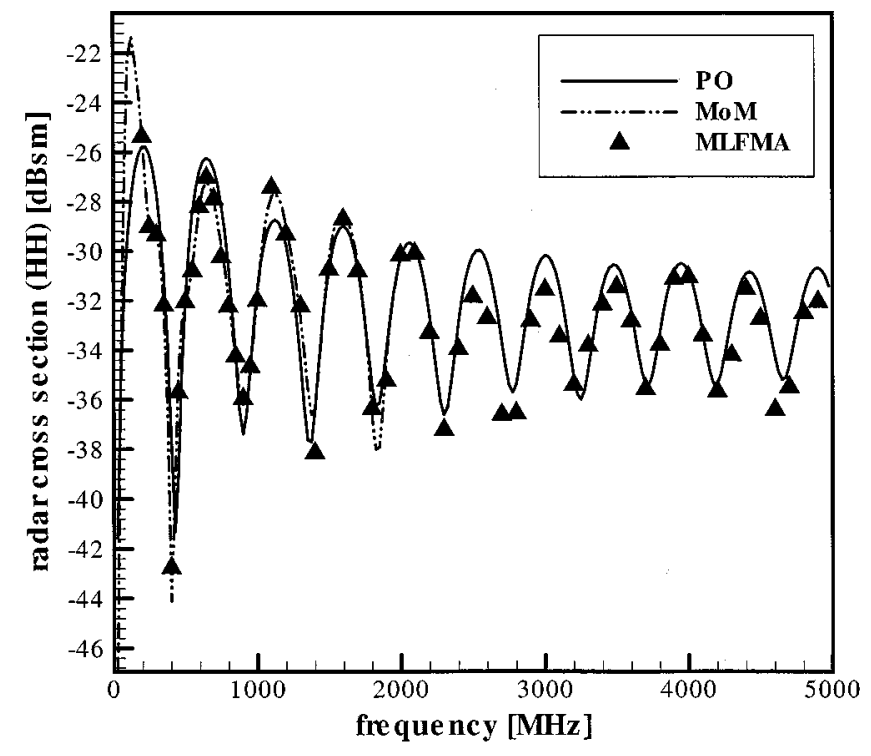

Fig. 3. As in Fig. 2, but for HH-polarization excitation and reception.

at approximately $1 \mathrm{~km} / \mathrm{h}$ while the basket is elevated to $45 \mathrm{~m}$ [14], [15]. For typical collection geometries, down-look angles to the target vary from $45^{\circ}$ to approximately $10^{\circ}$ (from grazing), depending on the range to the target and the height of the boom. The details of this fully polarimetric system are found in [14] and [15], as is a discussion on SAR-image formation.

\section{THEORETICAL AND MEASURED DATA}

\section{A. Comparison of Theoretical Models}

We first consider a direct comparison between the three theoretical models presented in Section II. In particular, we consider the 155-mm shell in Fig. 1 (155 mm maximum diameter). The 


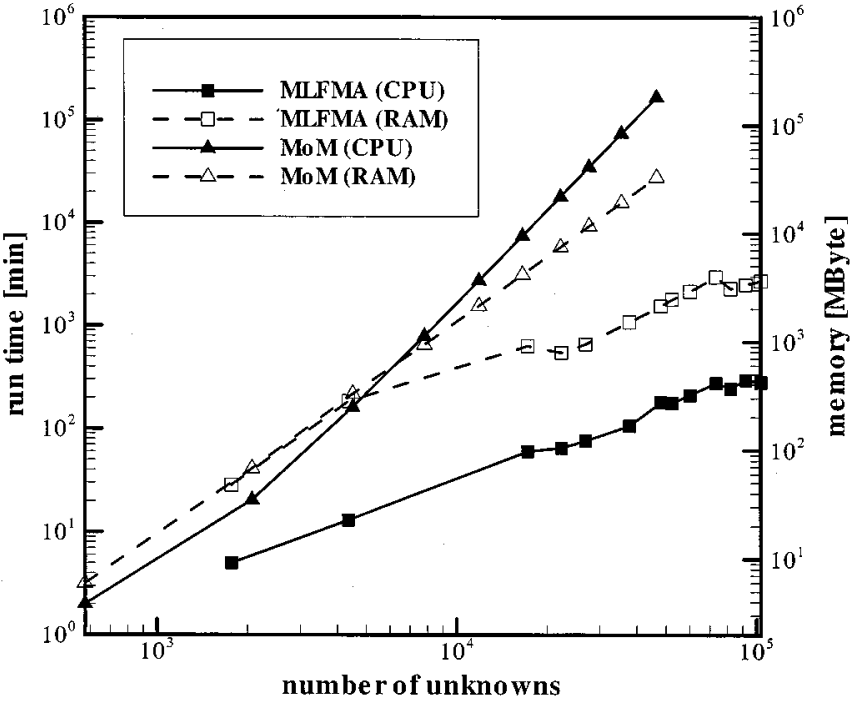

Fig. 4. For the results in Fig. 2, a comparison between the central processing unit (CPU) and memory (RAM) of the MoM and MLFMA results. The MoM results above $2 \mathrm{GHz}$ are extrapolated.



Fig. 5. Pulse shape and pulse spectrum (inset) for the time-domain plane-wave excitation used in the computed SAR images (Figs. 6 and 7).

length of the shell is $0.58 \mathrm{~m}$, representing nearly four free-space wavelengths at $2 \mathrm{GHz}$ (the buried target is of course even larger electrically). The length of the UXO is parallel to the air-ground interface, and the top of the UXO is $2.54 \mathrm{~cm}$ beneath the interface, the soil representative of Yuma soil with 5\% water content [15]. In Figs. 2 and 3 we plot the backscattered RCS for vertical $(\mathrm{VV})$ and horizontal $(\mathrm{HH})$ polarization, respectively, for incidence $30^{\circ}$ from grazing, directed $45^{\circ}$ azimuthally to the target broadside [see Fig. 6(b)]. In these results we plot data up to 5 $\mathrm{GHz}$, with the MLFMA and PO results plotted for all frequencies and the MoM results plotted only up to $2 \mathrm{GHz}$. The CPU and RAM requirements of the MoM are prohibitive when the target becomes electrically large, as it does above $2 \mathrm{GHz}$ (see Fig. 4). Hence, the MLFMA is used to examine the accuracy of PO when the target electrical size becomes large, corresponding to where the PO is expected to perform well.

The PO solution is in good agreement with the much more computationally expensive MoM and MLFMA solutions, for al- most all frequencies. The computation time of the PO results are infinitesimal (almost instantaneous) relative to MoM and MLFMA. With regard to the MoM and MLFMA solutions, the agreement between these models is almost exact, for all frequencies for which both are considered. However, as indicated in Fig. 4, the CPU and RAM requirements of the MoM are substantially higher than those of the MLFMA. In Fig. 4, for frequencies greater than $2 \mathrm{GHz}$, the MoM CPU and RAM are extrapolated from results at and below $2 \mathrm{GHz}$, assuming the expected order $N^{3}$ and $N^{2}$ variation, respectively (the MoM matrix equation was solved via a direct LU-decomposition, this of order $N^{3}$ complexity).

Close inspection of Figs. 2 and 3 reveals slight differences between the PO and MLFMA solutions, at frequencies above 2 GHz. This may appear somewhat anomalous, for the PO approximation is expected to become nearly exact at high frequencies. This latter expectation, however, is based on experience from free-space scattering. For the half-space problem, the PO solution does not account for multiple interactions between the target and the soil, regardless of the frequency. To this we attribute the small differences between the PO and MLFMA solutions at high frequencies. This shortcoming of the PO solution is examined further below, in the context of SAR images for the buried UXO. In Figs. 2 and 3 we only consider a single target-sensor orientation. In the SAR image we implicitly view the target from multiple orientations, and the aspect-dependent character of the signatures is also addressed more fully in Section IV.

\section{B. Measured and Computed SAR Imagery}

We compare the models to measurements by forming theoretical SAR images. All SAR-image examples below, both theoretical and experimental, are for VV polarization, approximately $30^{\circ}$ incidence from grazing. In computing the time-domain scattered waveform $s_{k}(t)$ from the $k$ th aperture position, we account for the $k$-dependent angle of incidence to a given image pixel, assuming plane wave excitation, pure polarization properties and a fixed incident-pulse shape (see Fig. 5). The latter two conditions are dictated by our incomplete knowledge of the antenna properties over ultrawide-band (UWB) frequencies for, in reality, the radiated pulse shape changes with variable target-antenna orientation, as does the polarization. On the other hand, the plane wave incidence approximation is typically appropriate for the SAR applications of interest since the distance between the sensor and targets is generally substantial. Finally, the waveforms $s_{k}(t)$ used to form the theoretical images are weighted as a function of aperture position, to approximately reflect the properties of the antenna pattern (see [15]).

The need to compute time-domain scattered waveforms at a large number of target-sensor orientations, for formation of a theoretical SAR image, places a significant burden on the computational requirements of the scattering model. In particular, the time-domain computations for each $s_{k}(t)$ used in the SAR image required 80 frequency calculations $(25-\mathrm{MHz}$ increments) over the 50-1200 MHz bandwidth of the experimental SAR system (from Fig. 5 we note that, while the usable SAR bandwidth is $50-1200 \mathrm{MHz}$, calculations are required up to $2 \mathrm{GHz}$ to cover the full bandwidth of the incident pulse). 




(a)

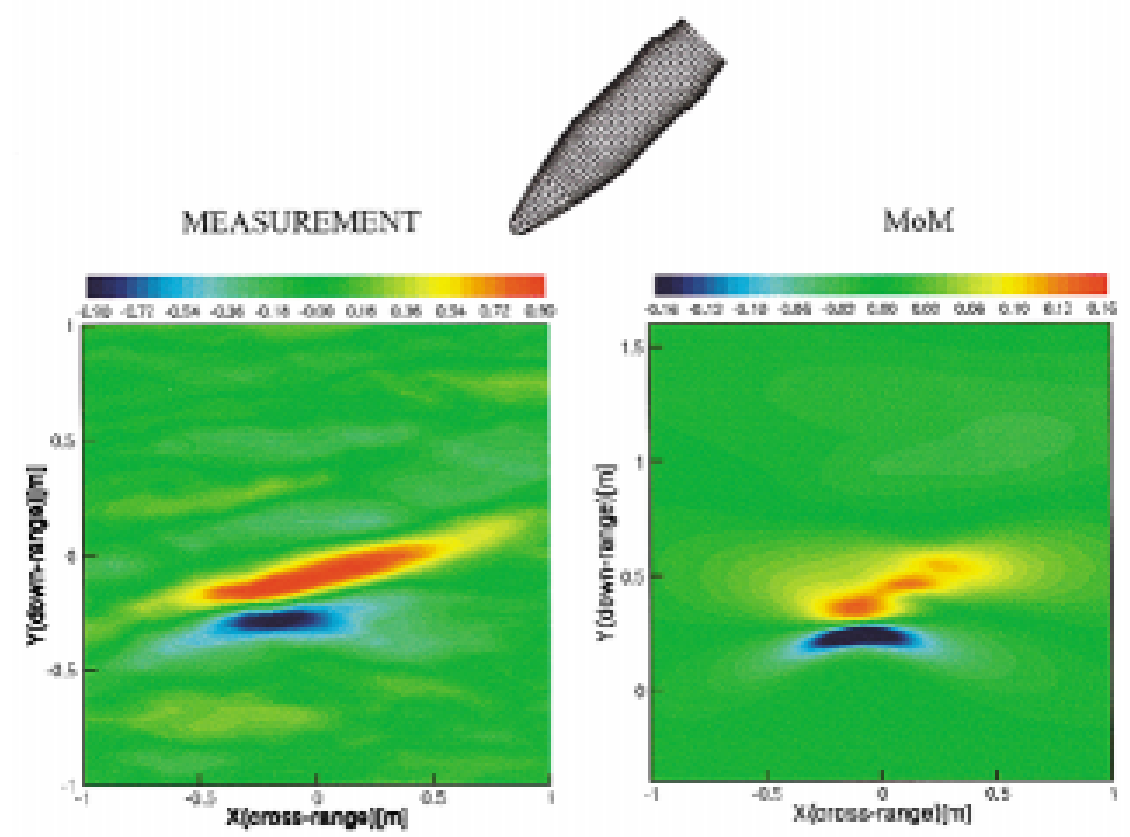

(b)

Fig. 6. Comparison of computed and measured SAR images for the 155-mm shell (Fig. 1) flush-buried in 5\% Yuma soil [15]. The computations use pulsed plane-wave excitation at $30^{\circ}$ from grazing, characterized by the pulse in Fig. 5 . The measured and computed images use an aperture length that yields a $60^{\circ}$ angle between the target center and aperture. (a) Shell axis parallel to linear SAR aperture. (b) Shell axis $45^{\circ}$ from linear SAR aperture.

Moreover, for computations of the SAR image, we employed an azimuthal sampling rate of $1^{\circ}$. We view the target from different azimuthal positions, as we traverse the linear SAR aperture and here we have precomputed time-domain scattered waveforms at a $1^{\circ}$ azimuthal sampling rate. Over the linear SAR aperture we must, in principle, also consider variation of the incident angle $\theta$, although for the aperture sizes considered this variation is negligible. Note that for rotationally symmetric land mines [12]-[14], [21], the target signature is independent of the azimuthal orientation, substantially reducing the number of scattering computations required to form the image [15]. The UXO half-space composite generally does not possess such symmetry. Consequently, in forming all the theoretical
SAR images presented below, we employ MoM and PO as the numerical forward solvers. If the MoM matrix equation is inverted via a direct LU-decomposition then, at a given frequency, multiple incident fields can be considered with little additional computational burden. By contrast, with the MLFMA the matrix equation is solved iteratively via the conjugate-gradient method [30]. In such iterative solvers, multiple incident fields must generally be computed separately, vitiating the use of MLFMA for theoretical SAR-image formation. We should note, however, that the MoM matrix multiplication is an order $N^{2}$ operation and for the image formation this must be done for all backscatter angles of interest (usually a large number). Therefore, overall, for the imaging problem 


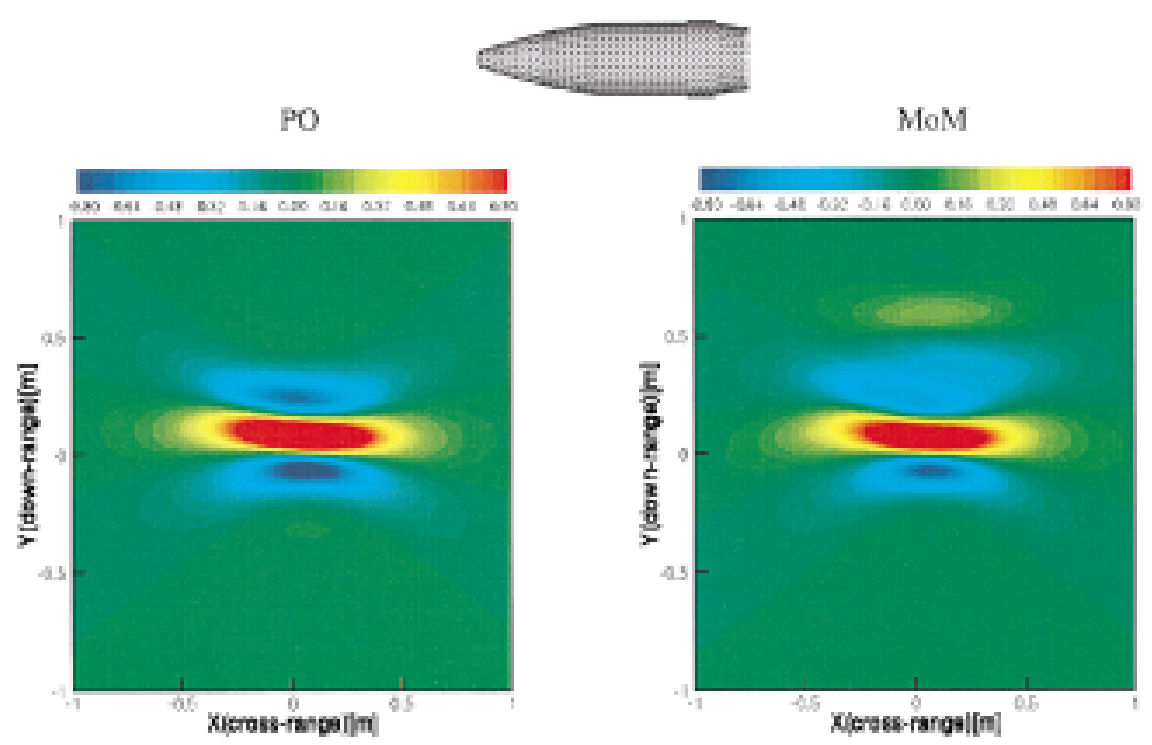

(a)

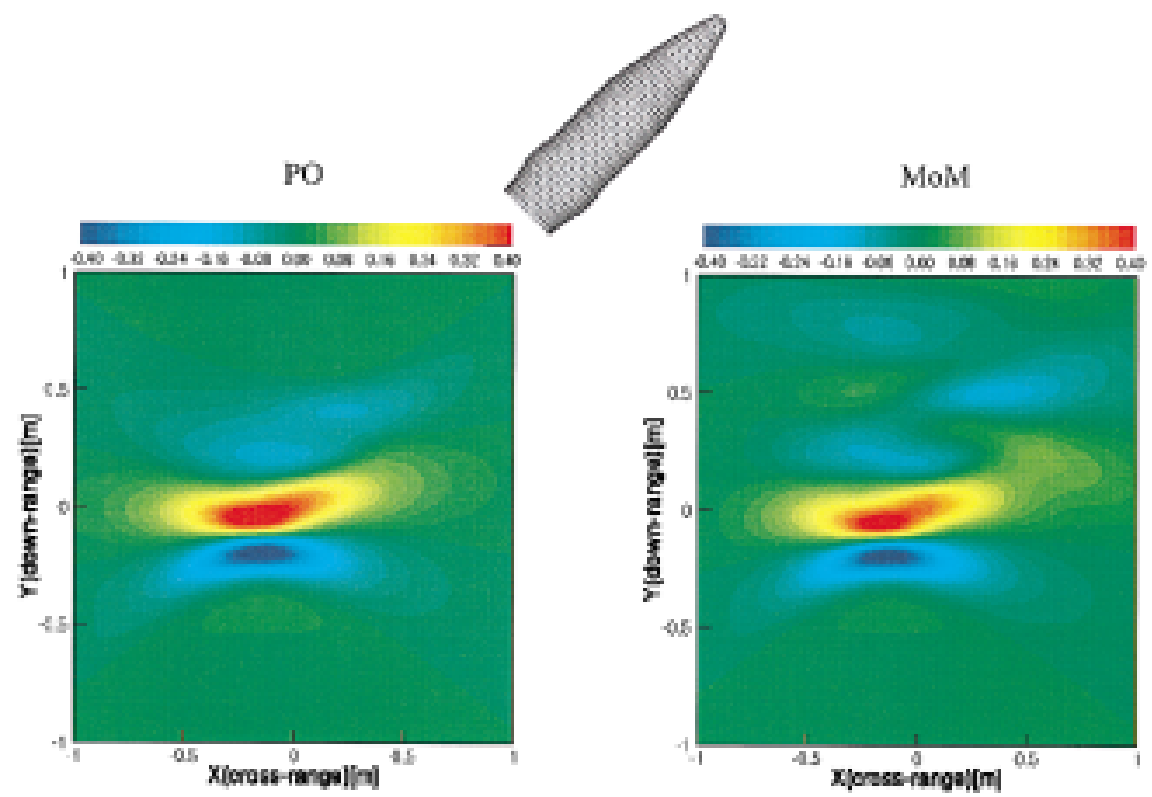

(b)

Fig. 7. Comparison of computed SAR images for the $155-\mathrm{mm}$ shell (Fig. 1) buried $2.54 \mathrm{~cm}$ in soil characterized by $\epsilon_{r}=5$ and $\sigma=0.003 \mathrm{~S} / \mathrm{m}$, with the target axis parallel to the air-soil interface. The computations use pulsed plane-wave excitation at $30^{\circ}$ from grazing, characterized by the pulse in Fig. 5.The images use an aperture length that yields a $60^{\circ}$ angle between the target center and aperture. The images are produced by scattered fields from MoM and PO forward solvers. (a) Incidence normal to the target axis. (b) Incidence $45^{\circ}$ from target axis.

of interest, MoM and MLFMA have similar computational burden when $N$ is large. In this context, the principal benefit of MLFMA vis-á-vis the MoM is in the area of RAM; the finite memory of a given computer limits the maximum target electrical size that can be considered, the MLFMA allowing consideration of significantly larger targets than the MoM (cf. Fig. 4).

The measured data is presented for the $155-\mathrm{mm}$ shell shown in Fig. 1, for a test site at Yuma Proving Ground in Yuma, AZ. The Yuma soil was characterized by approximately 5\% water content, with the associated electrical properties described in [15]. All measured results are for the UXO buried just under the air-ground interface, with the target axis parallel to the inter- face (flush buried). The gridded model of the target, used in the numerical computations, is shown in Fig. 6.

In Fig. 6(a) we consider the shell-oriented parallel to the linear SAR aperture, while in Fig. 6(b) the ordnance is oriented at $45^{\circ}$. For both images the total angle spanned by the linear aperture relative to the target center is $60^{\circ}$. The agreement between the model and measured SAR image is reasonable, especially considering the complexity of the experimental system and the uncertainty in the angle-dependent incident-wave polarization and pulse shape. As might be expected, when the target broadside is parallel to the linear aperture, the image is characterized primarily by a single strong return. Moreover, when tilted at $45^{\circ}$ the scattered return is characterized by two 

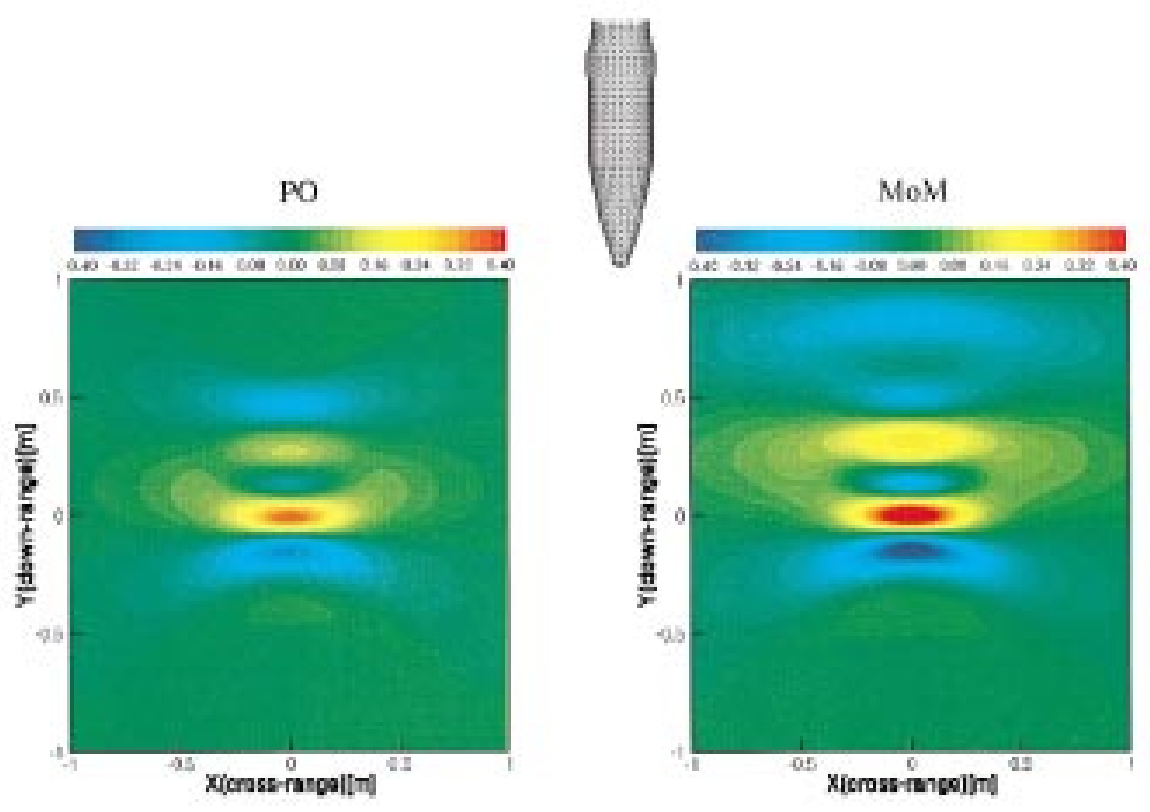

(c)

Fig. 7. (Continued.) Comparison of computed SAR images for the $155-\mathrm{mm}$ shell (Fig. 1) buried $2.54 \mathrm{~cm}$ in soil characterized by $\epsilon_{r}=5$ and $\sigma=0.003 \mathrm{~S} / \mathrm{m}$, with the target axis parallel to the air-soil interface. The computations use pulsed plane-wave excitation at $30^{\circ}$ from grazing, characterized by the pulse in Fig. 5.The images use an aperture length that yields a $60^{\circ}$ angle between the target center and aperture. The images are produced by scattered fields from MoM and PO forward solvers. (c) Incidence parallel to target axis.

diagonally offset returns, characteristic of diffraction from the target front and back. Note that, in the model results in Fig. 6(b), one can almost distinguish the two (diagonally offset) scattering mechanisms, while in the measured response these appear to merge into a single diagonal response. This may be due to an over estimation in the model of the SAR's cross-range resolution. Moreover, random motion of the sensor (for example, due to wind) also undermines the resolution of the measured image.

While the principal features of the measured and computed imagery (Fig. 6) are similar, there is a slight difference in the color scale of the images. This scaling difference is due to the fact that the experimental system is not calibrated, this constituting a very challenging task for the UWB system under consideration. Addressing this point further, to do system calibration, one requires knowledge of the theoretical response from fiducial targets to which the radar will be calibrated. Accurate models for the large fiducial targets of interest [20] is a nontrivial issue, it constituting a separate application of the MoM and MLFMA models.

While the theoretical SAR images in Fig. 6 capture most of the features in their measured counterparts, we notice several features in the measured images that are not seen in the computed data, this particularly true in Fig. 6(b). Fig. 6(b) is more sensitive to clutter because the target signature is driven by relatively weak edge diffraction, as compared to the specular reflection characteristic of Fig. 6(a). The image features not modeled by the theory are attributed to clutter, this, as discussed in Section I, constituting one of the principal challenges to SAR-based UXO detection (and why, as discussed further below, SAR is best for UXO near the soil interface). The measurements were taken in a relatively benign environment, with minimal foliage cover on the flat terrain. Consequently, the clutter in Fig. 6 is at- tributed primarily to subsurface soil inhomogeneities. It is very difficult to include such effects in a model for comparison to measured scattering data since the subsurface soil characteristics are often altered upon excavation (if due to localized soil moisture, for example). Clutter induced by subsurface inhomogeneities must be handled statistically, employing an appropriate statistical model for the subsurface soil properties.

Based on the accuracy of the PO results in Figs. 2 and 3, we next perform a comparison between a SAR image computed via $\mathrm{PO}$ with one computed via MoM, with both corresponding to the 155 -mm shell buried $2.54 \mathrm{~cm}$ under the Yuma-soil surface (5\% water content [15]), as in Figs. 2 and 3. We see in Fig. 7 that the agreement between the $\mathrm{PO}$ and MoM images is consistent with the comparisons in Figs. 2 and 3. We note, however, that there are noticeable small differences between the MoM and PO solutions. In particular, consider in Fig. 7(a) a linear cut in the image, running parallel to the vertical axis, through the image center (through the zero cross-range position). Increasing down-range corresponds to further distance between the target and sensor, and therefore increasing downrange in the image can also be viewed as increasing time (reflections viewed at the sensor at later times are represented in the image as being further from the sensor). Note that with increasing time (increasing downrange position), the MoM is characterized in Fig. 7(a) by a sequence of three bumps, followed by a faint fourth return. By contrast, the PO solution in Fig. 7(a), along the same cut, does not have this fourth faint return. This is explained as follows. The PO solution only includes the direct reflected waveform, with no multiple interaction between the buried target and the air-soil interface. By contrast, the rigorous MoM solutions should capture all phenomena, assuming sufficient numerical accuracy (e.g., enough basis functions). Consequently, the small differences between the PO and MoM solution in Fig. 7(a) are attributed to mul- 
tiple interaction between the target and the interface. Similar small differences are witnessed in Fig. 7(b) and (c). Nevertheless, these multiple interactions appear to constitute a small effect for this target. Such multiple interactions between the target and interface may also explain the small differences between the $\mathrm{PO}$ and rigorous solutions (MoM and MLFMA) seen in Figs. 2 and 3. Before leaving Fig. 7, it is important to emphasize that the PO solution captures most of the important shapes in the SAR image, relative to MoM. However, there is a noticeable difference in the amplitude of the PO solution, compared to MoM. We attribute this to the fact that the PO images use the PO forward solver at all frequencies, this being particularly inaccurate at the low frequencies of the incident pulse in Fig. 5, where there is considerable energy.

\section{Phenomenological Studies}

The principal goal of developing theoretical scattering models for surface and subsurface UXO is to gain a better understanding of the underlying wave phenomenology. We consider such phenomenological investigations below. Despite the fact that the PO model results in Sections IV-A-B looked promising, when performing phenomenological studies it is best to use a rigorous model, to be assured unexpected phenomena are witnessed, if present. Therefore, in the examples below, all data are computed via MoM and MLFMA.

The SAR imagery presented in the previous section indicates that the measured and computed SAR image for the target and soil considered is a function of the target orientation relative to the linearsynthetic aperture. Thisis a very important phenomenon, for it is such aspect-dependent UXO scattering that will help discriminate targets from clutter. To address this issue more completely, in Fig. 8 we plot the time-domain signature of the UXO target due to a pulsed plane wave of excitation in Fig. 5. The target is a 155 -mm shell buried as in Fig. 7 and here we plot the time-domain backscattered waveform as a function of target-sensor orientation. In particular, in Fig. 8, we plot the VV and $\mathrm{HH}$ time-domain scattered fields, for: 1) incidence normal to the target broad side; 2 ) at $45^{\circ}$ relative to broad side; and 3 ) along the target axis. The waveformsin 1)-3) are related toFig.7(a)-(c), respectively. These data were computed via the MoM model discussed in Section II-B. As expected, the time-domain signature is a strong function of orientation. The far-zone time-domain scattered waveforms in Fig. 8 are normalized by the distance $r$ between the target and observer and, therefore, the fields have units meters (analogous to square meters for RCS).

As expected, the broadside scattered waveform $\left(\phi=0^{\circ}\right)$ in Fig. 8 is significantly stronger than the other two angles, highlighting the distinction between primarily specular $\left(\phi=0^{\circ}\right)$ and edge-diffraction $\left(\phi=45^{\circ}\right.$ and $\left.\phi=90^{\circ}\right)$ scattering. Considering first the case $\phi=0^{\circ}$, we note the large specular return is followed by a considerably smaller second pulse (although that second pulse is nearly as large in amplitude as the scattered fields at $\phi=45^{\circ}$ and $\phi=90^{\circ}$ ). The second waveform for $\phi=0^{\circ}$ is attributed to interaction between the target and the air-ground interface since it is not seen for the PO results [see Fig. 7(a)]. The bandwidth is not sufficiently large to distinguish the specular return from diffraction from the two ends of the UXO, these two scattering mechanisms arriving nearly simulta-

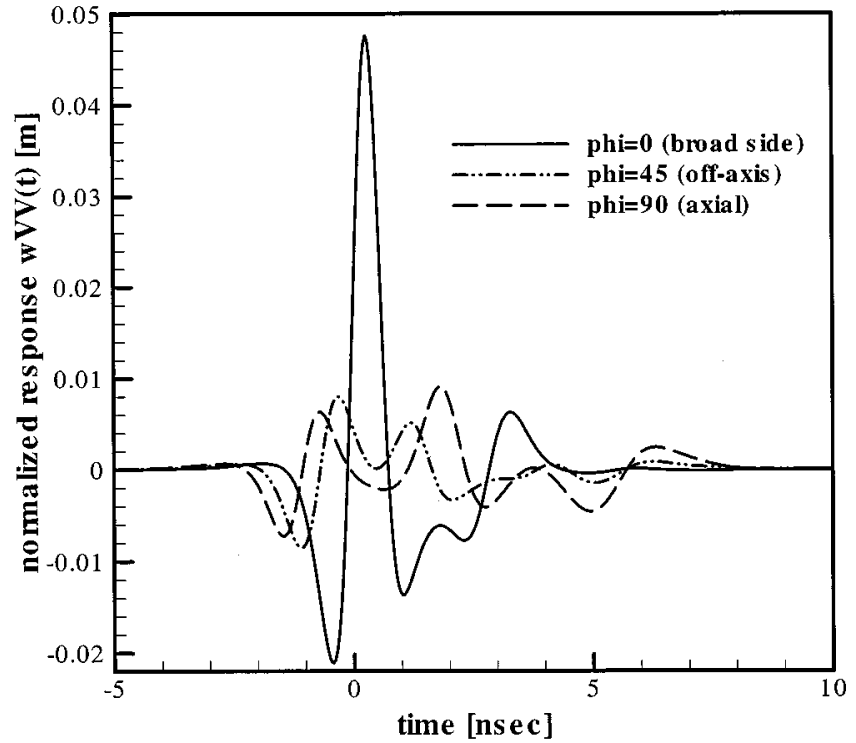

(a)



(b)

Fig. 8. Time-domain backscattered waveforms due to pulsed plane wave excitation at $30^{\circ}$ from grazing, with an incident pulse as in Fig. 5. The $155-\mathrm{mm}$ shell (Fig. 1) is buried $2.54 \mathrm{~cm}$ in 5\% Yuma soil [15], with its axis parallel to the air-soil interface. Considered are plane wave incidence normal to the target axis, $45^{\circ}$ from the target axis, and parallel to the target axis. (a) VV polarization. (b) $\mathrm{HH}$ polarization.

neously. Considering the other two incident angles $\left(\phi=45^{\circ}\right.$ and $\phi=90^{\circ}$ ), the scattered waveforms are characterized at early times by two consecutive scattered waveforms with, as expected, the duration between these waveforms larger for the case of $\phi=90^{\circ}$ than for $\phi=45^{\circ}$.

Note that the $\phi=0^{\circ}$ case is relevant for Fig. 6(a), for which the linear aperture yields scattering primarily from the target broadside. The fact that the $\phi=0^{\circ}$ signature is characterized principally by a single strong response explains the fact that Fig. 6(a) is also represented by a single strong return (in the image domain). Similarly, the two diffracted components for the $\phi=45^{\circ}$ case in Fig. 8 give rise to the two diffracted components characteristic of the image in Fig. 6(b), these apparently 


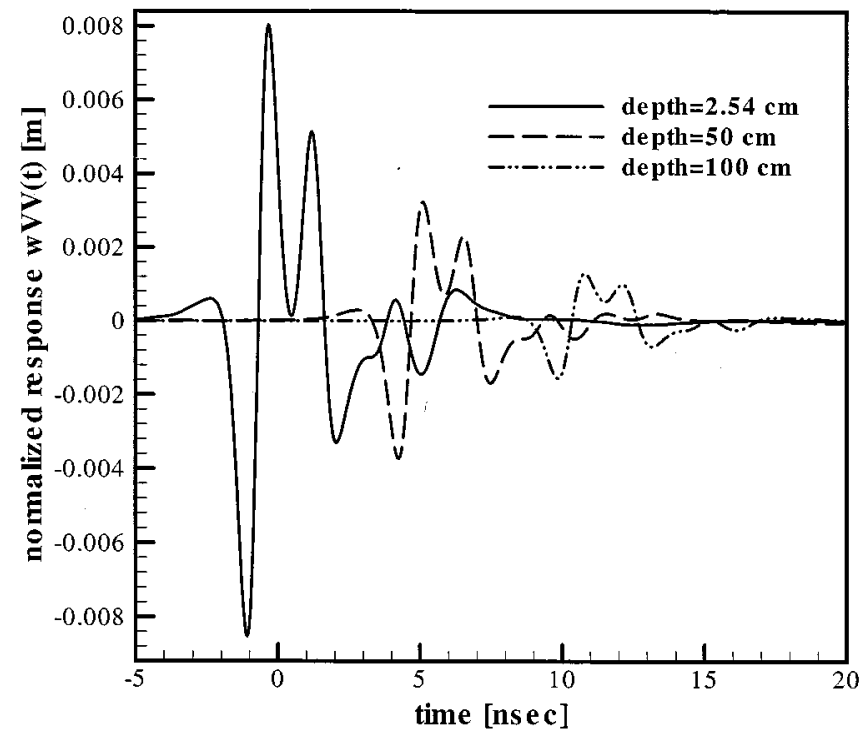

(a)

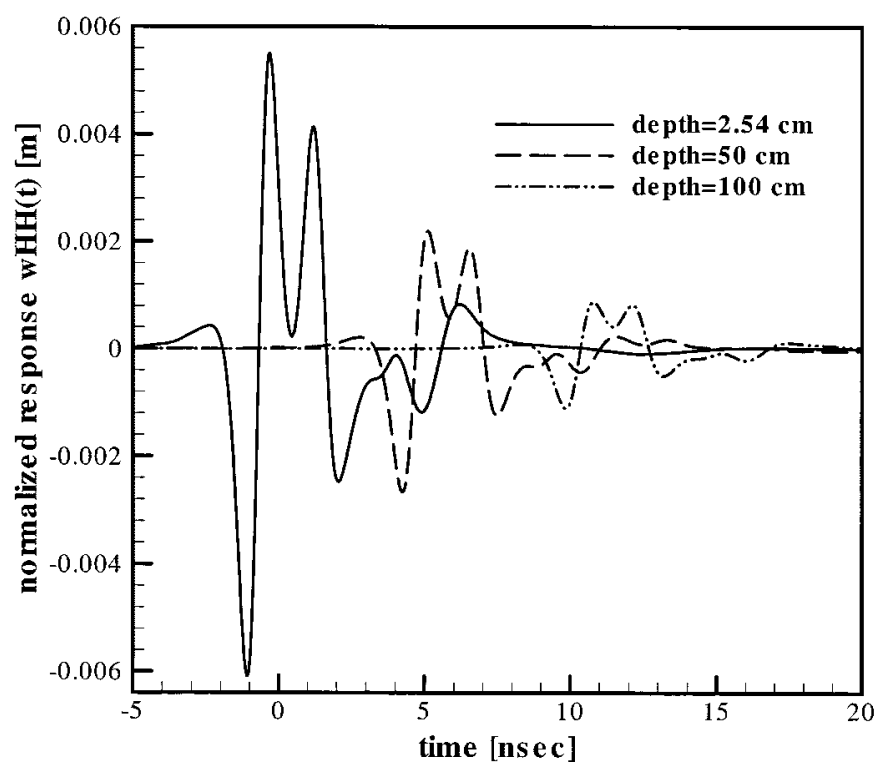

(b)

Fig. 9. Time-domain backscattered waveforms due to pulsed plane wave excitation $30^{\circ}$ from grazing, with an incident pulse as in Fig. 5. Three burial depths are considered for the 155-mm shell (Fig. 1): $2.54 \mathrm{~cm}, 50 \mathrm{~cm}$, and $100 \mathrm{~cm}$, with the target axis parallel to the air-soil interface (5\% Yuma soil [15]). The incident plane wave propagates $45^{\circ}$ from the target axis. (a) VV polarization. (b) $\mathrm{HH}$ polarization.

merging in the measured image into a single diagonal response. Moreover, the energy in the $\phi=0^{\circ}$ signature is significantly stronger than for $\phi=45^{\circ}$, this explaining why Fig. 6(b) is more contaminated by clutter than does Fig. 6(a).

In our next set of results, we again consider the $155-\mathrm{mm}$ shell buried in 5\% Yuma soil, but now we consider variable target depth. In particular, for simplicity, again assume that the UXO axis is parallel to the soil interface (the model is applicable to arbitrary target orientation [17], [22], [23]). We consider three target depths, all measured from the top of the target to the interface: 1) $2.54 \mathrm{~cm}$ as in Figs. 2 and 3; 2) $50 \mathrm{~cm}$; and 3) $100 \mathrm{~cm}$. We again consider time-domain plane-wave incidence, characterized by the pulse in Fig. 5 and all results are computed via the MLFMA. In Fig. 9, we present results for $30^{\circ}$ incidence relative to grazing, oriented $45^{\circ}$ relative to the target axis [as in Fig. 7(b)]. As expected, there is a significant reduction in the scattered-field amplitude with target depth. This phenomenon indicates that SAR-based sensing of UXO is of most utility for targets near the air-ground interface.

\section{CONCLUSION}

This paper has considered UWB scattering from UXO buried in lossy dispersive soil. Three scattering models were considered, and the numerical test considered here demonstrated that the MLFMA results are in close agreement with MoM for the buried UXO considered. Moreover, the agreement of the PO with the MoM/MLFMA solutions has been good, viewed both in the backscatter domain as well as in the SAR-image domain.

In addition to these theoretical investigations, we have performed SAR measurements via an experimental UWB sensor [15]. The agreement between theoretical and computed SAR images was good, despite uncertainty in the aspect-dependent radiated polarization and pulse shape. However, the clutter proved to be a significant issue, despite the fact that the UXO considered were shallow buried. The clutter was particularly problematic when the target orientation relative to the linear SAR aperture was such that no specular return was excited. In this case, the scattered fields are generated primarily by edge diffraction, the associated small scattered fields approaching the clutter level. We also demonstrated theoretically that this issue is exacerbated as the target depth increases. These theoretical and measured results indicate that SAR-based detection is most viable for detection of former bombing ranges, rather than for each individual (possibly deeply buried) UXO. This is motivated by the fact that most former ranges are littered by much surface and shallow-buried UXO. Once a former range is detected, alternative technology can be employed to attempt detection of each individual UXO [31].

The results presented here also indicate directions for future work. A principal concern in this context is gaining a better understanding of the mitigating clutter sources. For the UXO, the target shapes are generally well known (although the target is often deformed, due to impact) and, therefore, can be modeled. The clutter, on the other hand, is poorly understood. For example, the measured results presented here considered flat and weakly foliated terrain, but the SAR image still had clutter returns on the order of the shallow UXO, for the nonspecular case. The clutter source is conjectured to be subsurface soil inhomogeneties (e.g., spatially varying water content). Future work requires a combination of soil science and EM modeling to examine such issues in greater detail.

\section{REFERENCES}

[1] R. W. P. King and C. W. Harrison, "The transmission of electromagnetic waves and pulses into the earth," J. Appl. Phys., vol. 39, pp. 4444-4452, Aug. 1968.

[2] J. A. Fuller and J. R. Wait, "Electromagnetic pulse transmission in homogeneous dispersive rock," IEEE Trans. Antennas Propagat., vol. AP-20, pp. 530-533, July 1972.

[3] D. L. Moffat and R. J. Puskar, "A subsurface electromagnetic pulse radar,” Geophys., vol. 41, pp. 506-518, June 1976. 
[4] L. Peters and J. D. Young, "Applications of subsurface transient radars," in Time-Domain Measurements in Electromagnetics, E. K. Miller, Ed. New York: Van Nostrand Reinhold, 1986.

[5] G. S. Smith and W. R. Scott, "A scale model for studying ground penetrating radars," IEEE Trans. Geosci. Remote Sensing, vol. 27, pp. 358-363, July 1989.

[6] C. Liu and C. Shen, "Numerical simulation of subsurface radar for detecting buried pipes," IEEE Trans. Geosci. Remote Sensing, vol. 29, pp. 795-798, Sept. 1991.

[7] N. Osumi and K. Ueno, "Microwave holographic imaging of underground objects," IEEE Trans. Antennas Propagat., vol. AP-33, pp. 152-159, Feb. 1985.

[8] L. Peters, J. J. Daniels, and J. D. Young, "Ground penetrating radar as an environmental sensing tool," Proc. IEEE, vol. 82, pp. 1802-1822, Dec. 1994.

[9] J. M. Bourgeois and G. S. Smith, "A fully three-dimensional simulation of ground penetrating radar: FDTD theory compared with experiment," IEEE Trans. Geosci. Remote Sensing, vol. 34, pp. 36-48, Jan. 1996.

[10] S. F. Mahmoud and J. R. Wait, "Scattering from a buried insulated loop excited by an incident electromagnetic plane wave," J. Electromagn. Waves Applicat., vol. 12, pp. 1297-1311, Oct. 1998.

[11] B. A. Baertlein, J. R. Wait, and D. G. Dudley, "Scattering by a conducting strip over a lossy half space," Radio Sci., vol. 24, pp. 485-497, July/Aug. 1989.

[12] S. Vitebskiy, L. Carin, M. Ressler, and F. Le, "Ultrawide-band, shortpulse ground-penetrating radar: Simulation and measurement," IEEE Trans. Geosci. Remote Sensing, vol. 35, pp. 762-772, May 1997.

[13] L. Carin, R. Kapoor, and C. Baum, "Polarimetric SAR imaging of buried land mines," IEEE Trans. Geosci. Remote Sensing, vol. 36, pp. 1985-1988, Nov. 1998

[14] L. Nguyen, J. Sichina, K. Kappra, D. Wong, and R. Kapoor, "Minefield detection algorithm utilizing data from an ultra wideband wide-area surveillance radar," in Proc. 1998 SPIE Conf., Orlando, FL, Apr. 1998, pp. 627-643.

[15] L. Carin, N. Geng, M. McClure, J. Sichina, and L. Nguyen, "Ultrawide-band synthetic-aperture radar for mine-field detection," IEEE Antennas Propagat. Mag., vol. 41, pp. 18-33, Feb. 1999.

[16] J. K. Jao, "Performance analysis for synthetic aperture radar detection of underground targets," MIT Lincoln Laboratory, Lexington, MA, Project Rep. GPR-4, Oct. 1995.

[17] N. Geng, A. Sullivan, and L. Carin, "Multi-level fast-multipole algorithm for scattering from conducting targets above or embedded in a lossy half space," IEEE Trans. Geosci. Remote Sensing, vol. 38, pp. $1567-1579$, July 2000

[18] R. F. Harrington, Time-Harmonic Electromagnetic Fields. New York: McGraw-Hill, 1961.

[19] M. A. Ressler and J. W. McCorkle, "Evolution of the Army Research Laboratory ultra-wideband test bed," in Ultra-Wideband Short-Pulse Electromagnetics 2, L. Carin and L. B. Felsen, Eds. New York: Plenum, 1995, pp. 109-123.

[20] N. Geng, M. Ressler, and L. Carin, "Wideband VHF scattering from a trihedral reflector situated above a lossy dispersive halfspace," IEEE Trans. Geosci. Remote Sensing, vol. 37, pp. 2609-2617, Sept. 1999.

[21] N. Geng and L. Carin, "Wideband electromagnetic scattering form a dielectric BOR buried in a layered, dispersive medium," IEEE Trans. Antennas Propagat., vol. 47, pp. 610-619, Apr. 1999.

[22] N. Geng, A. Sullivan, and L. Carin, "Fast multipole method for scattering from 3-D PEC targets situated in a half-space environment," Microwave Opt. Tech. Lett., vol. 21, pp. 399-405, June 20, 1999.

[23] _ , "Fast multipole method analysis of half-space scattering problems," IEEE Trans. Antennas Propagat., to be published.

[24] R. Coifman, V. Rokhlin, and S. Wandzura, "The fast multipole method for the wave equation: A pedestrian prescription," IEEE Antennas Propagat. Mag., vol. 35, pp. 7-12, June 1993.

[25] J. M. Song and W. C. Chew, "Fast multipole method solution using parametric geometry," Microwave Opt. Tech. Lett., vol. 7, pp. 760-765, Nov. 1994.

[26] J. M. Song, C. C. Lu, and W. C. Chew, "Multilevel fast multipole algorithm for electromagnetic scattering by large complex objects," IEEE Trans. Antennas Propagat., vol. 45, pp. 1488-1493, Oct. 1997.

[27] A. F. Peterson, S. L. Ray, and R. Mittra, Computational Methods for Electromagnetics. Piscataway, NJ: IEEE Press, 1998.

[28] S. M. Rao, D. R. Wilton, and A. W. Glisson, "Electromagnetic scattering from surfaces of arbitrary shape," IEEE Trans. Antennas Propagat., vol. AP-30, pp. 409-418, May 1982.
[29] J. J. Yang, Y. L. Chow, and D. G. Fang, "Discrete complex images of a three-dimensional dipole above and within a lossy ground," Proc. Inst. Elect. Eng., pt. H, vol. 138, no. 4, pp. 319-326, Aug. 1991.

[30] T. K. Sarkar and E. Arvas, "On a class of finite step interative methods (conjugate directions) for the solution of an operator equation arising in electromagnetics," IEEE Trans. Antennas Propagat., vol. AP-33, pp. $1058-1066$, Oct. 1985.

[31] A. C. Dubey and R. L. Barnard, Eds., Detection and Remediation Technologies for Mines and Minelike Targets. Orlando, FL: Proc. SPIE, 1997, vol. 3079.

Anders Sullivan (M'93) was born August 9, 1963, in Staten Island, NY. He received the B.S. and M.S. degrees in aerospace engineering from the Georgia Institute of Technology, Atlanta, GA, in 1985 and 1987, respectively, and the Ph.D. degree in electromagnetics from Polytechnic University, Brooklyn, NY, in 1997.

From 1988 through 1998, he was with the Air Force Research Laboratory, Elgin Air Force Base, FL. From 1998 to 1999, he was with the Electrical Engineering Department, Duke University, Durham, NC, as a Research Associate. Since September 1999, he has been with the Army Research Laboratory, Adelphi, MD. His current research interests include modeling complex targets and short-pulse scattering.

Dr. Sullivan is a member of the Tau Beta Pi and Sigma Gamma Tau honor societies.

Raju Damarla (M'99) received the B.Sc.(mathematics), B.Tech. (electronics and electrical communication engineering), and M.Tech. (controls and computation engineering) degrees from the Indian Institute of Technology, Kharagpur, India, and the Ph.D. degree in engineering from Boston University, Boston, MA.

He was an Assistant Professor in the Electrical Engineering Department, University of Kentucky, Lexington, until 1994. He is currently working as an Electronics Engineer at the U.S. Army Research Laboratory, Adelphi, MD. His interests include signal processing, automatic target detection and recognition algorithm development, and neural networks.

Norbert Geng (S'91-M'96) was born May 14, 1965 in Lauchringen, Germany. $\mathrm{He}$ received the Dipl. Ing. and Dr. Ing. degrees in electrical engineering from the University of Karlsruhe, Germany, in 1991 and 1996, respectively.

From 1991 to 1996, he was with the Institute for Microwaves and Electronics at the University of Karlsruhe working on full-wave propagation modeling for radio communication systems. In January 1997, he joined the Department of Electrical and Computer Engineering, Duke University, Durham, NC, as a Visiting Postdoctor for 18 months. In July 1998, he returned to the University of Karlsruhe and, in 1999, he joined the Siemens Corporation, Munich, Germany. His current research interests include computational methods in electromagnetics and wave propagation modeling.

Dr. Geng received the Mannesmann Innovation Award in 1997.

Yanting Dong ( $\left.\mathrm{S}^{\prime} 00\right)$ received the B.S. and M.E. degrees in electrical engineering from Tianjin University, China, in 1993 and 1996, respectively. She is currently working toward the Ph.D. degree in the Department of Electrical and Computer Engineering, Duke University, Durham, NC.

From 1996 to 1998, she was with the Department of Automation, Tianjin University, China, working on process instrumentation design. Since 1999, she has been working as a Research Assistant at Duke University. Her main research interests are in signal processing, detection and estimation theory, applied electromagnetics, and instrumentation design. 
Lawrence Carin (SM'96) was born March 25, 1963, in Washington, DC. He received the B.S., M.S., and Ph.D. degrees in electrical engineering from the University of Maryland, College Park, in 1985, 1986, and 1989, respectively.

In 1989, he joined the Electrical Engineering Department, Polytechnic University, Brooklyn, NY, as an Assistant Professor and became an Associate Professor there in 1994. In September 1995, he joined the Electrical Engineering Department, Duke University, where he is an Associate Professor. His current research interests include short-pulse scattering, propagation, and signal processing. He is the Principal Investigator on an Army Research Office funded Multidisciplinary University Research Initiative (MURI) on demining.

Dr. Carin is a member of the Tau Beta Pi and Eta Kappa Nu honor societies. 